\title{
Differences in retinal vascular parameters between pediatric patients with inflammatory bowel diseases and healthy controls measured by optical coherence tomography angiography
}

\author{
Beata Klincewicz', Andrzej Michalski ${ }^{2}$, Marcin Stopa ${ }^{2}$ \\ 'Outpatient Department of Gastroenterology, Gastro Endo medic, Poznan, Poland \\ 2Department of Ophthalmology, Chair of Ophthalmology and Optometry, Poznan University of Medical Sciences, Poznan, Poland
}

\begin{abstract}
Introduction: Crohn's disease and ulcerative colitis, chronic inflammatory bowel diseases, can lead in some patients to extraintestinal manifestations within the eye. Due to coagulation abnormalities in the course of inflammatory bowel disease (IBD), severe impairment in retinal blood vessels and circulation can be present. Aim of the study: To assess the vascular network parameters in retinal and choroidal vessels with optical coherence tomography angiography (OCTA) in pediatric IBD patients and compare the obtained results with healthy controls.

Material and methods: Eighteen pediatric patients diagnosed with IBD in the remission state and 10 healthy controls were enrolled in the study. The near and distant visual acuity, cycloplegic refraction, intraocular pressure, and anterior and posterior eye segment were examined in every child. OCTA retina images in the $3 \times 3-\mathrm{mm}$ square centered on the fovea were obtained using the Optovue RTVue XR Avanti. The superficial capillary plexus layer (SL) and deep
\end{abstract}

capillary plexus layer (DL), and the size of the superficial (SFAZ) and deep foveal avascular zone (DFAZ) were calculated. Whole image, foveal and parafoveal vessel density for the superficial and deep plexus of the retina (SVD-Whole, SVD-Fovea, SVD-Pfovea; DVD-Whole, DVD-Fovea, DVD-Pfovea) were calculated. Results: A statistically significant difference was found in the foveal avascular zone (FAZ) area within the deep plexus. The vascular density in the whole image was significantly higher in the IBD group compared to the control group in the superficial plexus. The flow area in the superficial capillary plexus was increased considerably.

Conclusions: Superficial microvasculature parameters differ in pediatric IBD patients from healthy controls. The microvascular circulation in the posterior eye segment is changed in the course of IBD even though there is no clinical manifestation.

KEY WORDS: inflammatory bowel disease, OCT angiography, retina vasculature.

\section{INTRODUCTION}

Inflammatory bowel diseases (IBD) are regarded as chronic, lifelong disorders with exacerbations and remissions in their course. Two main types are distinguished: Crohn's disease (CD) and ulcerative colitis (UC). It has to be emphasized that about $25 \%$ of new cases of IBD are recognized in individuals before they reach 20 years of age [1-4]. Some patients experience extra-intestinal manifestations within the eye, such as lid swelling, blepharitis, conjunctivitis, episcleritis, anterior uveitis, chorioretinitis, corneal infiltrates, cataracts, choroiditis, retinal vasculitis, optic neuritis, serous retinal detachment and orbital pseudotumor. Different authors give their prevalence rate between $4 \%$ and 60\% [5-8]. Among described ophthalmological manifestations there are also ones associated with retinal circulation since the con- stantly active inflammatory process in IBD patients results in coagulation abnormalities (increased number of platelets, predisposition to thrombosis) [9]. In the case of thrombotic or embolic lesions, additional avascular zones were observed $[10,11]$. Therefore IBD is speculated to be a risk factor for serious abnormalities in retinal blood vessels and circulation.

For decades retinal vessels were pictured using fluorescein angiography. In this procedure, the intravenous contrast bolus enables retinal vessels' assessment [12]. In recent years optical coherence tomography angiography (OCTA), a noninvasive technique of retinal imaging, has been developed. It allows for visualization of both the retinal and choroidal vessels and is based on detection of the red blood cells' movement with new algorithms such as split-spectrum amplitude-decorrelation angiography (SSADA) $[13,14]$. Its significant advantage is 
the possibility not only to visualize but also to quantify vessels of either superficial or deep retinal plexuses. OCTA can detect pathological vessels in the retina and the layer of the choriocapillaris. Moreover, current software used in OCTA devices supports automatic measurement of the size of the superficial and deep foveal avascular zone and vessel density in the macular area. Many papers document this method's usability in children [15-18].

To the best of our knowledge, there are no results in the literature regarding the abnormalities in vascular density and foveal avascular zone parameters in children with IBD. For this reason our study aimed to assess the vascular network parameters in retinal and choroidal vessels with OCTA in pediatric IBD patients and compare the obtained results with healthy controls.

\section{MATERIAL AND METHODS}

\section{Study population}

The study had a prospective character and included pediatric patients diagnosed with IBD in the remission state, on maintenance treatment with the disease duration above five years. The clinical severity was classified based on the Pediatric Ulcerative Colitis Activity Index (PUCAI) and Pediatric Crohn's Disease Activity Index (PCDAI). Both for PUCAI and PCDAI the result was below 10 points. Patients were excluded if: any other severe chronic diseases such as asthma, cystic fibrosis, kidney insufficiency, congenital heart failures or any ocular disorders including high myopia were present. The control group consisted of healthy children matched with age. The presented study received the approval of the Bioethics Committee at Poznań University of Medical Sciences (Ref. 436/2016) and was conducted between May 2016 and January 2017.

\section{Data acquisition and image analysis}

The near and distant visual acuity, cycloplegic refraction, intraocular pressure, and anterior and posterior eye segment were examined in every child. After that OCTA retina images in the $3 \times 3-\mathrm{mm}$ square centered on the fovea were obtained using the Optovue RTVue XR Avanti. The OCTA examination was conducted twice, and the average of the results was taken for statistical analysis. The built-in AngioVue Analytics software automated algorithms (RTVue_XR2017.0.0.16) segmented the superficial capillary plexus layer (SL) and deep capillary plexus layer (DL) automatically. Afterward the same automated algorithms were used to calculate the area of the superficial (SFAZ) and deep foveal avascular zone (DFAZ). Whole image, foveal and parafoveal vessel density for the superficial and deep plexus of the retina (SVDWhole, SVD-Fovea, SVD-Pfovea; DVD-Whole, DVD-Fovea, DVD-Pfovea) were automatically calculated. The parafovea was defined as an annulus centered on the fovea with inner and outer ring diameters of 1 and $3 \mathrm{~mm}$ respectively. We measured flow area in $\mathrm{mm}^{2}$ in the circle of $1 \mathrm{~mm}$ diameter centered on the fovea (Flow-SP, Flow-DP, Flow-CC). An experienced ophthalmologist assessed accurate scan centering.
Data were evaluated only if the signal strength index (SSI) was 75 or above.

\section{Statistics}

All results are expressed as the mean \pm standard deviation. We examined the variables for normal distribution with the Kolmogorov-Smirnov and Shapiro-Wilk tests. The measurements in the study and control groups were compared with the Mann-Whitney test. A p-value less than 0.05 was defined as statistically significant. The calculations were performed using the Statistica program (StatSoft).

\section{RESULTS}

\section{Patient characteristics}

This study evaluated 18 pediatric patients (36 eyes) with IBD, treated using either immunosuppressants or mesalazine, drugs that have no vasodilatative activity. All subjects were Caucasians. Mean age of the patients was $14.0 \pm 1.78$ (range 11-17 years). The control group consisted of 10 healthy children (20 eyes) with mean age $14.5 \pm 2.87$ (range 8-17 years). The best corrected visual acuity ranged from 0.05 to -0.1 logMAR (Table I). The cycloplegic refraction in both groups was in the range from $-0.50 \mathrm{D}$ to $+1.50 \mathrm{D}$ sphere, and up to $0.75 \mathrm{D}$ cylinder. No significant difference in the baseline demographics, which included age, gender and best-corrected visual acuity, was found between the study and the control group. We found that imaging with no artifacts and with the SSI of at least 75 was generally possible in children aged 8 and older.

\section{Foveal avascular zone area}

The mean FAZ area in the superficial plexus was 0.253 $\pm 0.094 \mathrm{~mm}^{2}$ in the IBD group and $0.229 \pm 0.056 \mathrm{~mm}^{2}$ in the control group. The difference was not statistically significant $(p=0.3030)$. The mean FAZ area in the deep plexus was $0.335 \pm 0.089 \mathrm{~mm}^{2}$ in the IBD group and $0.406 \pm 0.135 \mathrm{~mm}^{2}$ in the control group. The difference was statistically significant $(p=0.0202)$.

\section{Vessel density parameters}

The vascular density in the whole image was significantly higher in the IBD group compared to the control group in the superficial plexus ( $p=0.0135$ ), but not in the deep plexus $(p=0.9795)$. The sector analysis showed a statistically significant difference between groups for parafoveal vessel density in the superficial plexus only ( $p=0.0074)$ (Tables II and III).

Table I. Group characteristics

\begin{tabular}{|l|c|c|}
\hline Parameter & Examined group $(n=18)$ & Control group $(n=10)$ \\
\hline Age (years) & $14.0 \pm 1.78$ & $14.5 \pm 2.87$ \\
\hline Males & $56 \%(10)$ & $50 \%(5)$ \\
Females & $44 \%(8)$ & $50 \%(5)$ \\
\hline Ulcerative colitis & 9 & 0 \\
Crohn's disease & 9 & 0 \\
\hline Best corrected & $-0.05 \pm 0.10$ & $-0.04 \pm 0.11$ \\
visual acuity & & \\
\hline
\end{tabular}


Table II. Vessel density parameters - mean values

\begin{tabular}{|l|c|c|c|c|c|c|} 
& SVD-Whole & SVD-Fovea & SVD-Pfovea & DVD-Whole & DVD-Fovea & DVD-Pfovea \\
\hline Examined group & $53.20 \pm 2.59$ & $34.03 \pm 5.37$ & $55.07 \pm 2.55$ & $58.64 \pm 2.09$ & $31.51 \pm 5.65$ & $61.92 \pm 2.03$ \\
\hline Control group & $51.11 \pm 3.07$ & $34.11 \pm 3.98$ & $52.86 \pm 3.02$ & $58.65 \pm 1.95$ & $30.42 \pm 4.34$ & $62.45 \pm 1.67$ \\
\hline$p$ & 0.0135 & 0.8642 & 0.0074 & 0.9795 & 0.5103 & 0.1533 \\
\hline
\end{tabular}

For superficial retina plexus: SVD-Whole - whole image vessel density, SVD-Fovea - foveal vessel density, SVD-Pfovea - parafoveal vessel density; and for deep plexus of the retina: DVD-Whole - whole image vessel density, DVD-Fovea - foveal vessel density, DVD-Pfovea - parafoveal vessel density (values given in \%)

Table III. Vessel density parameters - median and interquartile range

\begin{tabular}{|c|c|c|c|c|c|c|}
\hline & SVD-Whole & SVD-Fovea & SVD-Pfovea & DVD-Whole & DVD-Fovea & DVD-Pfovea \\
\hline \multirow[t]{2}{*}{ Examined group } & 52.87 & 35.32 & 54.82 & 59.16 & 32.15 & 62.21 \\
\hline & 2.91 & 7.15 & 3.38 & 3.04 & 7.94 & 2.42 \\
\hline \multirow[t]{2}{*}{ Control group } & 51.32 & 33.87 & 52.91 & 59.13 & 30.95 & 62.93 \\
\hline & 3.08 & 5.35 & 3.07 & 1.72 & 5.18 & 1.72 \\
\hline
\end{tabular}

Table IV. Flow area (in $\mathrm{mm}^{2}$ )

\begin{tabular}{|l|c|c|c|} 
& Flow-SP & Flow-DP & Flow-CC \\
\hline Examined group & $1.450 \pm 0.099$ & $1.515 \pm 0.101$ & $1.917 \pm 0.053$ \\
\hline Control group & $1.360 \pm 0.134$ & $1.444 \pm 0.140$ & $1.882 \pm 0.064$ \\
\hline$p$ & 0.0235 & 0.0726 & 0.0635 \\
\hline
\end{tabular}

Flow-SP - for superficial plexus of the retina, Flow-DP - for deep plexus of the retina, Flow-CC - for choriocapillaris.

Flow area in superficial, deep microvasculature and choriocapillaris

There was no statistically significant difference in flow area between the IBD group and control group in the deep capillary plexus and choriocapillaris (Table IV). Nevertheless, the flow area in the superficial capillary plexus was significantly higher in the examined group $(p=0.0235)$.

\section{The absence of pathological vasculature}

Retinal and choroidal OCTA images in the $3 \times 3-\mathrm{mm}$ square centered on the fovea did not reveal any pathological neovascularization. Abnormal vessels were not present in eyes from IBD and control groups.

\section{DISCUSSION}

This study demonstrates for the first time that the vascular density and foveal flow area in the superficial capillary plexus were significantly higher in the IBD group compared to the control group. Interestingly, vessel density and foveal flow area in the deep capillary plexus did not differ between the groups. However, FAZ in the deep capillary plexus was larger in the control group. Furthermore, flow area in the choriocapillaris was comparable in children from both groups.

Extra-intestinal manifestations in the course of IBD are described in $25 \%$ to $40 \%$ of patients. The most common signs appear in the joints, skin, liver and biliary tract, but also within the eye. Data from the medical literature have shown that the eye complications emerge in the majority of cases in patients with CD limited to the large bowel and terminal ileum. Apart from long-lasting disease, young age for disease onset and involvement of distant organs increase the risk of eye complications. It is postulated that arthritis in patients with $\mathrm{CD}$ raises the risk of occurrence of inflammatory lesions in the eye by $33 \%$ [19]. Eye complications involve mainly the anterior segment with such conditions as scleritis and keratitis. Reports on abnormalities in the posterior eye segment are scarce. There were published case reports only describing both adult and pediatric patients. Falavarjani et al. described a 9-year old boy with CD and recognized thrombosis of the retinal artery [20]. Trojet et al. reported the case of a 55-year old man with retinal vasculitis [21].

It is speculated that thrombotic and occlusive pathomechanisms play a crucial role in the development of lesions in the posterior eye segment. The basis of underlying mechanisms is associated with lymphocytes infiltration of perivascular spaces, vessel endothelium injuries, and prothrombin hyperactivity [22-24]. Ghanchi concluded that almost half of the patients with IBD, in the course of the disease, experience at least one episode of extra-intestinal manifestations in the eye. In some cases, the eye abnormalities can precede classic gastrological symptoms of IBD. Based on patients' observation, eye complications can be assumed as independent from the extent of inflammatory lesions within the alimentary tract [9]. Interestingly, they usually appear in early years of IBD duration [25]. According to Manganelli et al., 18\% of patients with IBD can have asymptomatic eye lesions [26]. The prospective study conducted by Felekis et al. confirms these data. The authors examined 60 persons with IBD. They diagnosed eye lesions in the anterior or posterior segment in $43 \%$ of patients. It is worth mentioning that none of the patients had a clinical demonstration [27]. Our observations correspond to the Felekis results - none of the children presented clinical symptoms from the eye. In our study the vessel density for the superficial plexus was statistically significantly different for vascularity both in the whole image and parafoveally. For selected flow area statistical significance was found for the superficial plexus. The obtained results allowed us to conclude 
that functioning of the circulatory system was impaired in the course of IBD. We hypothesize that peripheral microvasculature was modified by the raised concentration of inflammatory mediators that change the vessel endothelium activity. Schinzari et al. demonstrated the influence of TNF- $\alpha$ and vascular endothelial growth factor (VEGF) on the vascular endothelium in patients with IBD [28]. In the course of IBD, the changes in the blood components and vessel wall structure result in blood flow impairment. It was demonstrated in Caliscan's paper [29]. The retrobulbar blood flow velocities and resistive indices of retrobulbar arteries in patients with $\mathrm{CD}$ were examined. The results were correlated with findings of healthy individuals (control group). No significant differences in flow parameters of the posterior ciliary artery were found between the two groups. Peak systolic velocity of the central retinal artery was higher in patients with $C D$, which correlates with results obtained in our study for superficial vessel density (for the whole image and parafoveal region), which was statistically higher in the study group. The difference in end diastolic velocity and resistive indices showed no statistical significance. For CD patients end-diastolic velocity of the ophthalmic artery was lower than for the control group. Resistive indices of the ophthalmic artery were higher for patients with CD, and peak systolic flow velocities were similar in both groups. Additionally, carotid intima-media thickness is a parameter connected with cardiovascular risk, and it was thicker in patients with Crohn's disease. Based on study outcomes, the authors concluded that higher resistive indices of the ophthalmic artery correlate with increased carotid intimamedia thickness. It shows that retrobulbar blood flow examination may be a diagnostic tool for detection of microvascular dysfunction in patients with CD [29]. We hypothesize that OCTA could be used in the future to evaluate the cardiovascular risk by the measurement of increased blood flow in the superficial capillary plexus.

Keles et al. described exciting findings. In his study, retrobulbar blood flow velocities and resistivity index measurement were performed for patients with chronic inflammatory disease (renal amyloidosis) and compared to the control group. They did not observe any statistically significant differences in parameters of blood flow in the central retinal artery. However, peak systolic flow velocity of the posterior ciliary artery was significantly higher in the study group. The end diastolic velocity of the ophthalmic artery of patients with renal amyloidosis was substantially lower and the resistivity index higher than in the control group [30]. The mentioned study, like ours, captures very subtle changes in blood flow parameters. Therefore, it indicates that in chronic diseases microvascular impairment is present.

OCTA is a noninvasive method and, as the literature shows, can be successfully used in the pediatric population aged 8 and above [31]. It is noteworthy that there exist no clear recommendations and guidelines for eye care in IBD patients [30]. As the risk for permanent sight impairment exists due to vascular damage in the eyeball, we hypothesize that vascular and choroidal perfusion parameters measured with OCTA could be employed in monitoring and early detection of vascular complications within the eye in children with IBD.

The limitations of this study included the limited field of OCTA imaging. The small scanning area is a significant shortcoming of current OCTA systems. It is possible that vascular changes can be present outside of the $3 \times 3$ scanning area. This issue can be addressed in the future with OCTA devices with a wider field of view. The relatively small sample size may be a limitation of this study given the decreased statistical power. Another limitation which should be considered is lack of axial length $(\mathrm{AL})$ measurement and proper recalculation of obtained results. Decrease of superficial plexus vessel density and enlarged FAZ in myopic children were described by Gołębiewska et al. [32], but we examined only children with low refractive error. However, we did not measure AL, which is variable even in an emmetropic population. The influence of AL on results of automated measurements and the mathematical correction formula was described in a paper by Sampson et al. [33].

We showed that superficial microvasculature parameters differ in pediatric IBD patients from healthy controls. It indicates that microvascular circulation in the posterior eye segment is changed in the course of IBD even though there is no clinical manifestation. However, future studies are required.

\section{DISCLOSURE}

The authors declare no conflict of interest.

\section{References}

1. Virta L, Saarinen MM, Kolho KL. Inflammatory bowel disease incidence is on the continuous rise among all paediatric patients except for the very young: a nationwide registry-based study on 28-year follow-up. J Crohns Colitis 2017; 11: 150-156.

2. Malmborg $P$, Hildebrand $H$. The emerging global epidemic of paediatric inflammatory bowel disease - causes and consequences. J Intern Med 2016; 279: 241-258.

3. Buderus $S, S c h o l z S$, Behrens R, et al. Inflammatory bowel disease in pediatric patients: Characteristics of newly diagnosed patients from the CEDATA-GPGE Registry. Dtsch Arztebl Int 2015; 112: 121-127.

4. Benchimol EL, Bernstein CN, Bitton A, et al. Trends in epidemiology of pediatric inflammatory bowel disease in Canada: distributed network analysis of multiple population-based provincial health administrative databases. Am J Gastroenterol 2017; 112: 1120-1134.

5. Karmiris K, Avgerinos A, Tavernaraki A, et al. Prevalence and characteristics of extra-intestinal manifestations in a large cohort of Greek patientswith inflammatory bowel disease. J Crohns Colitis 2016; 10: 429-436.

6. Felekis T, Katsanos K, Kitsanou M, et al. Spectrum and frequency of ophthalmologic manifestations in patients with inflammatory bowel disease: a prospective single-center study. Inflamm Bowel Dis 2009; 15: 29-34. 
7. Mintz R, Feller ER, Bahr RL, Shah SA. Ocular manifestations of inflammatory bowel disease. Inflamm Bowel Dis 2004; 10: 135-139.

8. Yilmaz $S$, Aydemir $E$, Maden A, Unsal B. The prevalence of ocular involvement in patients with inflammatory bowel disease. Int J Colorectal Dis 2007; 22: 1027-1030.

9. Ghanchi DF, Rembacken BJ. Inflammatory bowel disease and the eye. Surv 0phthalmol 2003; 48: 663-676.

10. De Carlo TE, Romano A, Waheed N, et al. A review of optical coherence tomography angiography (OCTA). Int J Retina Vitreous 2015; 1:5.

11. Gao SS, Jia Y, Zhang M, et al. Optical coherence tomography angiography. Invest Ophthalmol Vis Sci 2016; 57: 0CT27-36

12. Novotny HR, Alvis DL. A method of photographing fluorescence in circulating blood in the human retina. Circulation 1961;24:82-86.

13. Tokayer J, Jia Y, Dhalla AH, et al. Blood flow velocity quantification using split-spectrum amplitude-decorrelation angiography with optical coherence tomography. Biomed Opt Express 2013; 4: 1909-1924.

14. Jia Y, Tan 0, Tokayer J, et al. Split-spectrum amplitude-decorrelation angiography with optical coherence tomography. Opt Express 2012; 20: 4710-4725.

15. Gołębiewska J, Olechowski A, Wysocka-Mincewicz M, et al. Optical coherence tomography angiography vessel density in children with type 1 diabetes. PLoS One 2017; 12: e0186479.

16. Zhang Z, Huang X, Meng X, et al. In vivo assessment of macula in eyes of healthy children 8 to 16 years old using optical coherence tomography angiography. Sci Rep 2017; 7: 8936.

17. Chen X, Viehland C, Carrasco-Zevallos OM, et al. Microscope-integrated optical coherence tomography angiography in the operating room in young children with retinal vascular disease. JAMA Ophthalmol 2017; 135: 483-486.

18. Molnar A, Holmström G, Larsson E. Macular thickness assessed with spectral domain OCT in a population-based study of children: normative data, repeatability and reproducibility and comparison with time domain OCT. Acta Ophthalmol 2015; 93: 470-475.

19. Siqueira RC, Kaiser RL, Ruiz LP, et al. Ischemic retinopathy associated with Crohn's disease. Int Med Case Rep J 2016; 9: 197-200.

20. Falavarjani KG, Parvaresh MM, Shahraki K, et al. Central retinal artery occlusion in Crohn disease. J AAPOS 2012; 16: 392-393.

21. Trojet S, Mazlout H, Hamdouni M, et al. Retinal vasculitis in Crohn's disease: a case report. J Fr Ophtalmol 2010; 33: 54-55.

22. Das KM. Relationship of extraintestinal involvements in inflammatory bowel disease: new insights into autoimmune pathogenesis. Dig Dis Sci 1999; 44: 1-13.

23. Ha C, Magowan S, Accortt NA, et al. Risk of arterial thrombotic events in inflammatory bowel disease. Am J Gastroenterol 2009; 104: 1445-1451.

24. Kappelman MD, Horvath-Pubo E, Sandler RS, et al. Thromboembolic risk among Danish children and adults with inflammatory bowel diseases: a population-based nationwide study. Gut 2011; 60: 937-943.

25. Hopkins DJ, Horan E, Burton IL, et al. Ocular disorders in a series of 332 patients with Crohn's disease. Br J Ophthalmol 1974; 58: 732-737.

26. Manganelli C, Turco S, Balestrazzi E. Ophthalmological aspects of IBD. Eur Rev Med Pharmacol Sci 2009; 13 Suppl 1: 11-13.

27. Felekis T, Katsanos K, Kitsanou M, et al. Spectrum and frequency of ophthalmologic manifestations in patients with inflammatory bowel disease: a prospective single-center study. Inflamm Bowel Dis 2009; 15: 29-34.

28. Schinzari F, Armuzzi A, De Pascalis B, et al. Tumor necrosis factor-alpha antagonism improves endothelial dysfunction in patients with Crohn's disease. Clin Pharmacol Ther 2008; 83: 70-76.

29. Caliskan Z, Keles N, Kahraman R, et al. Imparied retrobulbar blood flow and increased carotid IMT in patients with Crohn's disease. Int J Cardiovasc Imaging 2016; 32: 1617-1623.

30. Keles N, Caliskan M, Aksu FU, et al. Retrobulbar blood flow and carotid intima-media thickness alteration may relate to subclinic atherosclerosis in patients with chronic inflammatory diseases. Ren Fail 2015; 37: 1164-1170.

31. Ottaviano G, Moretti A, Luini C, et al. Which concern about ocular involvement in IBD pediatric patients? Dig Liver Dis 2016; 48: e271.

32. Zhang Z, Huang $X$, Meng $X$, et al. In vivo assessment of macula in eyes of healthy children 8 to 16 years old using optical coherence tomography angiography. Sci Rep 2017; 7: 8936.

33. Gołębiewska J, Biała-Gosek K, Czeszyk A, Hautz W. Optical coherence tomography angiography of superficial retinal vessel density and foveal avascular zone in myopic children. PLoS One 2019; 14: e0219785.

34. Sampson DM, Gong P, An D, et al. Axial length variation impacts on superficial retinal vessel density and foveal avascular zone area measurements using optical coherence tomography angiography. Invest Ophthalmol Vis Sci 2017; 58: 3065-3072. 